\title{
Perioperative cardiac risk assessment in kidney transplantation: It's time to search for a new gold standard
}

\author{
Yasmeen Golzar, MD, ${ }^{a}$ and Rami Doukky, MD, MSc, MBA, FASNC ${ }^{\mathrm{a}, \mathrm{b}}$ \\ a Division of Cardiology, Cook County Health, Chicago, IL \\ b Division of Cardiology, Rush University Medical Center, Chicago, IL
}

Received May 9, 2021; accepted May 10, 2021

doi: $10.1007 / \mathrm{s} 12350-021-02673-\mathrm{z}$

\section{See related article, pp. 3405-3415}

Increased cardiac risk associated with advanced chronic kidney disease (CKD) poses a particular challenge when evaluating candidates for renal transplantation. The incidence of myocardial infarction (MI) in renal transplant candidates is considerable, ranging $8.7 \%-16.7 \%$ by 3 years after transplant listing and $4.7 \%-11.1 \%$ after transplantation. ${ }^{1}$ Alarmingly, almost half of the deaths that occur within 30 days after kidney transplantation are due to $\mathrm{MI}^{2}$ With little data to help guide cardiac risk stratification and even fewer tools to mitigate that risk, not only do transplant teams have to contend with a challenging peri-transplant period, they must also grapple with how to ethically, yet prudently allocate scarce donor organs in an extraordinarily high-risk patient population.

Identifying reliable methods to assess cardiac risk is the first step in working toward effectively decreasing peri-transplant cardiac event rates. Unfortunately, guideline-based risk assessment tools used in the general population do not perform well when applied to this uniquely high-risk patient population. ${ }^{3,4}$ While current perioperative guidelines rely on symptom-limited exercise capacity to guide risk stratification, the majority of end-stage renal disease (ESRD) patients who have significant coronary artery disease (CAD) do not display typical symptoms. ${ }^{9}$ Hence, the 2012 American Heart

Reprint requests: Rami Doukky, MD, MSc, MBA, FASNC, Division of Cardiology, Cook County Health, 1901 W. Harrison Street, Chicago, IL 60612; rdoukky@cookcountyhhs.org

J Nucl Cardiol 2022;29:3416-8.

$1071-3581 / \$ 34.00$

Copyright (C) 2021 American Society of Nuclear Cardiology.
Association (AHA)/American College of Cardiology (ACC) consensus statement on cardiac disease evaluation and management among kidney and liver transplantation candidates veers away from standard practice by proposing the use of non-invasive CAD surveillance in asymptomatic patients with three or more pre-defined risk factors (age $>60$ years, hypertension, diabetes, cardiovascular disease, dyslipidemia, smoking, dialysis $>1$ year, and left ventricular hypertrophy). ${ }^{3,4}$ Whether this screening approach improves patient survival or transplant outcomes is yet to be determined. ${ }^{4}$

Without strong evidence to guide this practice, the question remains: Does an aggressive approach to stress testing asymptomatic kidney transplant candidates truly help to identify occult, high risk, and (ideally) modifiable CAD to drive down perioperative cardiac event rates, or does it just drive away potential transplant candidates by unreasonably delaying or excluding those who have an abnormal non-invasive test? Part of the answer lies in the diagnostic and prognostic performance of available non-invasive imaging tools. Stress singlephoton emission computed tomography (SPECT) myocardial perfusion imaging (MPI) is the most commonly used stress testing modality in CKD patients. Along with its prognostic value being well established in this patient population, ${ }^{5-8}$ several studies specifically evaluating the prognostic utility of stress SPECT-MPI in renal transplant candidates have shown that an abnormal MPI is a predictor of adverse cardiac events. ${ }^{9,10}$ However, data from mostly small, single-site studies suggest that the diagnostic performance of SPECT-MPI in this patient population may be less reliable.

In this issue of the Journal, Kelderman et al. present an updated comprehensive meta-analysis assessing the diagnostic accuracy of SPECT-MPI for detection of significant CAD in patients being evaluated for kidney transplantation. ${ }^{11}$ They analyzed data from 13 studies, including a total of 1245 SPECT scans compared to the 
"gold standard" of invasive coronary angiography. SPECT-MPI studies, including the use of variable radioisotopes and acquisition protocols, were designated as abnormal based on the presence of fixed or reversible perfusion defects or the calculated summed stress score. The presence of CAD had variable definitions based on percentage of stenosis of epicardial coronary arteries (five studies used $\geq 50 \%$ stenosis, six studies used $\geq$ $70 \%$ stenosis, and two studies used a combination of $\geq$ $50 \%$ and $\geq 70 \%$ stenosis). Pooled results showed that SPECT-MPI had a sensitivity of $66 \%$ and specificity of $75 \%$. These findings led the authors to conclude that SPECT-MPI has only moderate diagnostic accuracy in candidates being evaluated for kidney transplantation. ${ }^{11}$

Despite the variability in radioisotopes, SPECT-MPI protocols, and angiographic definitions of CAD used in the included studies, this meta-analysis offers a valuable re-examination of the limited diagnostic utility of SPECTMPI in renal transplant candidates. Even with the inclusion of more contemporary studies, the authors' findings are very similar to previously published pooled results which delineate the suboptimal diagnostic performance of SPECT-MPI in detecting significant macrovascular CAD in kidney transplant candidates. ${ }^{3,9,12}$

In acknowledging the limitation of using a functional imaging test against the anatomic "gold standard" of coronary angiography as inherently problematic, the authors touch on an important point. Our current risk stratification strategies rely primarily on the premise of detecting and intervening on high-risk macrovascular CAD. Given our burgeoning understanding that the presence of myocardial ischemia and anatomic macrovascular CAD are not mutually inclusive, this unidimensional approach to perioperative risk assessment is worthy of re-examination.

Ultimately, the value of a preoperative risk assessment and disease detection tool is in its ability to (1) accurately predict perioperative outcomes, and (2) diagnose risk-modifying disease, the treatment of which can be used to improve survival. On both fronts, coronary angiography is an imperfect "gold standard." Angiographically defined coronary stenosis has not been shown to be a reliable prognostic tool, having variable correlation with subsequent clinical events in renal transplant candidates. While some observational studies have reported worse outcomes in patients with angiographic $\mathrm{CAD}$, others have shown that this increased risk only exists in specific patient populations such as those with proximal CAD or with nondiabetic kidney failure. Furthermore, recent studies have found no correlation between the presence of significant CAD on angiography and patient survival. ${ }^{13}$

The fact that macrovascular CAD is not a reliable predictor of cardiac event rates reflects the complex and multifactorial mechanism of perioperative MI. While patients with significant macrovascular CAD are at higher risk of perioperative MI by the mechanism of demand ischemia, the phenomenon of plaque rupture is unpredictable and most often occurs in non-flow limiting coronary lesions which would go undetected in stress testing and would not warrant intervention even if detected on coronary angiography. Moreover, patients with advanced kidney disease have impaired coronary microcirculation and decreased coronary flow reserve. ${ }^{3}$ In the perioperative period, impaired coronary flow dynamics likely contribute to the high risk of ischemic events seen in renal transplant patients, even in the absence of macrovascular CAD.

Positron emission tomography (PET), with its ability to assess myocardial blood flow and coronary flow reserve, is a promising non-invasive tool which is able to integrate the hemodynamic effects of epicardial coronary stenosis and microvascular dysfunction. ${ }^{14}$ Though PET-MPI has not been studied specifically in renal transplant candidates, it has been shown to be an effective prognostic tool in CKD and ESRD patients as an independent and incremental predictor of adverse cardiac outcomes. ${ }^{14,15}$ Future research is needed to better understand the potential role of PET-MPI in the cardiac evaluation of renal transplant candidates. However, no matter how well-performing a prognostic device may be, risk stratification strategies will only function to be exclusionary tools unless they are coupled with effective risk reduction strategies, of which we have very few. With a new "gold standard," it is imperative that we simultaneously work toward effective interventions that will help improve peri-transplant outcomes. Only then, will we truly be able to better serve this high-risk patient population.

\section{Disclosures}

The authors have indicated that they have no financial conflict of interest.

\section{References}

1. Manoushagian S, Meshkov A. Evaluation of solid organ transplant candidates for coronary artery disease. Am J Transplant 2014; $14: 2228-34$

2. Knoll G, Cockfield S, Blydt-Hansen T, Baran D, Kiberd B, Landsberg D et al. Canadian Society of Transplantation consensus guidelines on eligibility for kidney transplantation. CMAJ 2005; 173:1181-84

3. Wang LW, Fahim MA, Hayen A, Mitchell RL, Lord SW, Baines LA et al. Cardiac testing for coronary artery disease in potential kidney transplant recipients: A systematic review of test accuracy studies. Am J Kidney Dis 2011; 57:476-87 
4. Doukky R, Fughhi I, Campagnoli T, Wassouf M, Kharouta M, Vij A et al. Validation of a clinical pathway to assess asymptomatic renal transplant candidates using myocardial perfusion imaging. J Nucl Cardiol 2018; 25:2058-68

5. Golzar Y, Doukky R. Stress SPECT myocardial perfusion imaging in end-stage renal disease. Curr Cardiovasc Imaging Rep 2017; 10:1-13

6. Vij A, Golzar Y, Doukky R. Regadenoson use in chronic kidney disease and end-stage renal disease: A focused review. J Nucl Cardiol 2018; 25:137-49

7. Doukky R, Fughhi I, Campagnoli T, Wassouf M, Ali A. The prognostic value of regadenoson SPECT myocardial perfusion imaging in patients with end-stage renal disease. J Nucl Cardiol 2017; 24:112-8

8. Kolkailah AA, Iskander M, Iskander F, Patel PP, Khan R, Doukky R. The prognostic utility of regadenoson SPECT myocardial perfusion imaging in patients with end-stage renal disease: The largest cohort to date. J Nucl Cardiol 2020. https://doi.org/10.10 07/s12350-020-02259-1

9. Kassab K, Doukky R. Cardiac imaging for the assessment of patients being evaluated for kidney transplantation. J Nucl Cardiol. 2021. https://doi.org/10.1007/s12350-021-02561-6

10. Cantoni V, Green R, Acampa W, Assante R, Zampella E, Nappi C et al. Prognostic value of myocardial perfusion imaging in patients with chronic kidney disease: A systematic review and meta-analysis. J Nucl Cardiol. 2021. https://doi.org/10.1007/s12350-02002449-x
11. Kelderman J, Jolink F, Benjamens S, Monroy-Gonzalez A, Pol R, Slart R. Diagnostic accuracy of myocardial perfusion imaging in patients evaluated for kidney transplantation: A systematic review and meta-analysis. J Nucl Cardiol. 2021. https://doi.org/10.1007/ s12350-021-02621-x

12. Parikh K, Appis A, Doukky R. Cardiac imaging for the assessment of patients being evaluated for kidney or liver transplantation. $\mathrm{J}$ Nucl Cardiol 2015; 22:282-96

13. Lentine KL, Costa SP, Weir MR, Robb JF, Fleisher LA, Kasiske BL et al. Cardiac disease evaluation and management among kidney and liver transplantation candidates: A scientific statement from the American Heart Association and the American College of Cardiology Foundation. J Am Coll Cardiol 2012; 60:434-80

14. Shah NR, Charytan DM, Murthy VL, Skali Lami H, Veeranna V, Cheezum MK et al. Prognostic value of coronary flow reserve in patients with dialysis-dependent ESRD. J Am Soc Nephrol 2016; 27:1823-29

15. Murthy VL, Naya M, Foster CR, Hainer J, Gaber M, Dorbala S et al. Coronary vascular dysfunction and prognosis in patients with chronic kidney disease. JACC Cardiovasc Imaging 2012; 5:102534

Publisher's Note Springer Nature remains neutral with regard to jurisdictional claims in published maps and institutional affiliations. 